\title{
Adsorción en la Vida Cotidiana. Un Experimento de Fisicoquímica con Aplicación Ambiental
}

\author{
Virginia Savy, Oriana D'Alessandro, Graciela M. Valle, Laura E. Briand \\ Universidad Nacional de La Plata, Facultad de Ciencias Exactas, Centro de Investigación y \\ Desarrollo en Ciencias Aplicadas Dr. J. J. Ronco, Centro Científico Tecnológico La Plata, \\ CONICET. Calle 47 No 257, B1900AJK La Plata, Buenos Aires-Argentina. \\ (e-mail: briand@quimica.unlp.edu.ar)
}

Recibido Sep. 28, 2011; Aceptado Nov. 03, 2011; Versión final recibida Dic. 11, 2011

\begin{abstract}
Resumen
Este trabajo presenta un enfoque innovador para estudiar el fenómeno de adsorción teniendo en cuenta la perspectiva ambiental. En este contexto se estudiaron las fisisorciones de dos sustancias comunes, ácido acético e hipoclorito de sodio, sobre carbón activado como un caso típico de tratamiento de contaminantes en fase acuosa. Para ello se estudiaron las cinéticas y las isotermas de adsorción a temperatura ambiente. Estos experimentos involucran una serie de técnicas básicas de laboratorio, como las titulaciones ácido-base y redox, el tratamiento de datos, su representación gráfica y el ajuste de los resultados experimentales con los modelos de adsorción de Langmuir y de Freundlich. Se muestra finalmente que el trabajo experimental permite que los alumnos adquieran habilidades de laboratorio de química con experimentos simples.
\end{abstract}

Palabras clave: enseñanza de la química, química experimental, adsorción, cinética, carbón activado

\section{Adsorption in Everyday Life. A Physicochemical Experiment with Environmental Application}

\begin{abstract}
An innovative approach to study the fundamentals of adsorption phenomena considering an environmental perspective is presented. In this context, the physisorption of two common substances, acetic acid and sodium hypochlorite, over activated carbon was chosen as a case study of a typical remediation process of water contaminants. The physisorption phenomenon was investigated by determining the process kinetics and the adsorption isotherms at room temperature. These experiments involve a series of basic laboratory techniques such as acid-base and redox titrations, along with data manipulation, graphical presentation and calculations to fit the experimental data to the models of Langmuir and of Freundlich. Finally, it is shown that the experimental work allows students to acquire laboratory abilities with simple experiments.
\end{abstract}

Keywords: chemistry teaching, experimental chemistry, adsorption, kinetics, activated carbon 


\section{INTRODUCCIÓN}

La industria química y petroquímica genera aguas residuales que contienen contaminantes orgánicos poco amigables con el medio ambiente, como es el caso de los pertenecientes a la familia de los fenoles $\left(\mathrm{C}_{6} \mathrm{H}_{5} \mathrm{OH}\right)$. Se han desarrollado muchas técnicas para su descomposición a dióxido de carbono $\left(\mathrm{CO}_{2}\right)$ y agua $\left(\mathrm{H}_{2} \mathrm{O}\right)$, pero lamentablemente, a veces mediante esta descomposición se generan compuestos refractarios, como es el caso de los ácidos de cadena corta como el ácido acético $\left(\mathrm{CH}_{3} \mathrm{COOH}\right)$. Aunque este ácido es difícil de degradar, su separación del medio acuoso puede realizarse mediante adsorción con carbón activado (Cuizano et al., 2009). Por otra parte, en los tratamientos convencionales de aguas residuales se realiza una cloración como pretratamiento químico, confiriéndole sabor al agua debido a la presencia de hipoclorito, el que se puede separar también con carbón activado. Los filtros de carbón activado $\left(C^{*}\right)$ tienen la capacidad de adherir ciertas sustancias a su superficie (adsorción) y por lo tanto, se utilizan para la remoción de sustancias orgánicas, inorgánicas y biológicas que generan color, olor y gusto (Dhawale, 1993). En la actualidad muchos hogares cuentan con "filtros o jarras filtradoras" en cuyo interior uno de sus componentes suele ser $C^{*}$. Este hecho cotidiano brinda una atractiva oportunidad para plantear a los alumnos la necesidad de comprender qué es lo que sucede cuando utilizamos estos dispositivos al tomar agua en nuestros hogares (Jacobsen, 2008).

En este contexto, la adsorción física (fisisorción) de sustancias sobre carbón activado puede asociarse a una pelota de tenis que rebota sobre una superficie hasta que pierde energía y se acomoda en algún sitio no específico. Las moléculas adsorbidas mantienen su integridad aunque pueden estar levemente distorsionadas por acción de la superficie del adsorbente. Una vez alcanzado el equilibrio no hay variación de la concentración del adsorbato en la superficie del adsorbente ni en la solución.

Una vez conocido el tiempo en el cual se alcanza el equilibrio es posible la realización de las isotermas de adsorción las cuales representan la distribución en equilibrio de un adsorbato entre un sólido y una fase líquida a temperatura constante. En la práctica, las isotermas de adsorción se representan a través de la cantidad del soluto (adsorbato) que se encuentra adsorbido por unidad de masa de adsorbente (Cads/m) versus la concentración del adsorbato en equilibrio (Cequ.).

El presente trabajo propone una metodología experimental para transmitir conocimientos relacionados con la química de superficies, la problemática de la contaminación del agua y su remediación a través de métodos físicos. En particular se utiliza una temática de gran actualidad con el fin de captar la atención de los alumnos universitarios, estimular su participación en el proceso de construcción del conocimiento (D'Alessandro et al., 2009) y brindar herramientas para resolver futuros problemas medioambientales.

\section{METODOLOGÍA}

El carbón se coloca en un recipiente de vidrio (de peso conocido) y se activa a través del calentamiento a la llama del mechero hasta que se elimina completamente la humedad. El recipiente se cierra, se deja enfriar hasta temperatura ambiente y se pesa para determinar la masa del carbón anhidro.

Para la realización de las cinéticas de adsorción se ponen en contacto $200 \mathrm{mg} \mathrm{C*}$ con $50.00 \mathrm{~mL}$ de solución de concentración conocida de ácido acético (Anedra, 99.7\%) denominado de ahora en adelante HAc y de hipoclorito de sodio (lavandina comercial, $50 \mathrm{~g} / \mathrm{L} \mathrm{Cl}$ ) respectivamente, a temperatura ambiente y bajo agitación magnética. La toma de muestra se realiza cada 5 minutos durante 50 minutos, la determinación de la cantidad adsorbida se realiza por titulación de la cantidad remanente y de esta manera se establece el tiempo necesario para alcanzar el equilibrio.

Conocido el tiempo de contacto para alcanzar el equilibrio, se realizan las isotermas de adsorción para cada sistema. Se toman 6 frascos y en cada uno se ponen en contacto el $C^{*}$ y las diferentes concentraciones iniciales de adsorbato a temperatura constante y agitación homogénea. Transcurrido el tiempo necesario para alcanzar el equilibrio se procede a la toma de muestras. 
La concentración de ácido acético se determinó por medio de una titulación ácido-base convencional con hidróxido de sodio $(\mathrm{NaOH})$ de concentración conocida. Asimismo, la determinación de la concentración de $\mathrm{NaClO}$ se realizó por titulación redox con tiosulfato de sodio $\left(\mathrm{Na}_{2} \mathrm{~S}_{2} \mathrm{O}_{3}\right)$ (Celsi y Copello, 1960). Las titulaciones se realizaron por duplicado.

Los datos se pueden ajustar a través de los modelos propuestos por Langmuir o Freundlich, entre otros, lo que permite obtener más información acerca de las características del sistema adsorbatoadsorbente en estudio. Las formas lineales para las isotermas de Langmuir y Freundlich se expresan mediante las ecuaciones (1) y (2) respectivamente,

$\frac{1}{(\text { Cads } / m)}=\frac{1}{x K} \frac{1}{\text { Cequ }}+\frac{1}{x}$

$\log ($ Cads $/ m)=\log K_{f}+\frac{1}{n_{f}} \log$ Cequ

donde $x$ es la máxima cantidad de soluto para formar una monocapa en la superficie, $m$ es la masa de adsorbente y $K, K_{f}$ y $n_{f}$ son constantes características del sistema.

Mediante las representaciones de: $1 /(\mathrm{Cads} / \mathrm{m})$ vs $1 /$ Cequ y log (Cads $/ \mathrm{m})$ vs. log Cequ, es posible determinar los valores de las constantes y a qué modelo se ajustan los resultados obtenidos. Si el factor de correlación lineal $(r)$ es cercano a la unidad podemos decir que los datos se ajustan a tal o cual modelo y a partir de los valores de ordenada y pendiente se pueden determinar los valores de las constantes características. El factor de correlación junto con los valores de pendiente y ordenada se pueden determinar a través de un programa del tipo hoja de cálculo como el Excel.

\section{RESULTADOS Y DISCUSIÓN}

De las cinéticas de adsorción resulta que el tiempo necesario para alcanzar el equilibrio es de 20 min. para el HAc de concentración inicial $\mathrm{C}_{0}=0.0612 \mathrm{M}$ y de $50 \mathrm{~min}$. para el $\mathrm{NaClO}$ de concentración inicial $\mathrm{C}_{0}=0.88$ \% p/v como se puede ver en la Figura 1.
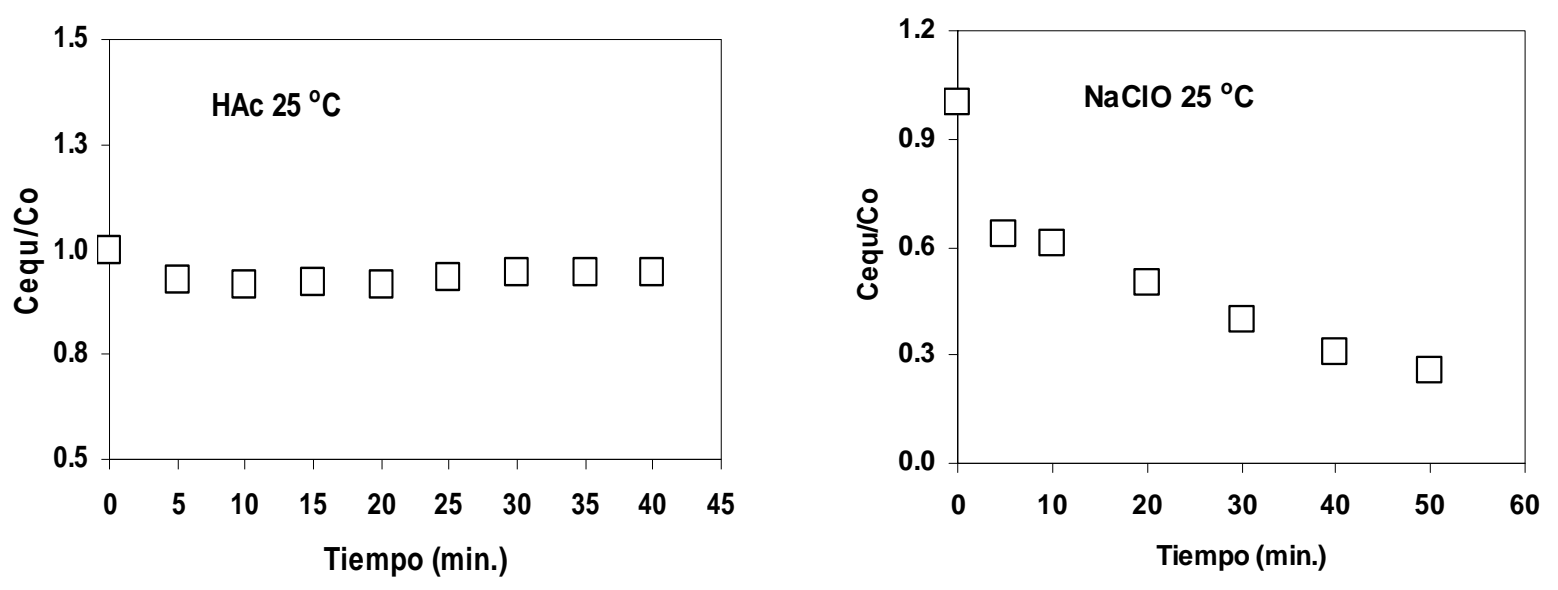

Fig. 1: cinéticas de adsorción para HAc y $\mathrm{NaClO}$.

De las isotermas de adsorción resulta que ambos sistemas adsorbato-adsorbente se ajustan al modelo Freundlich como se puede ver en la Figura 2. 

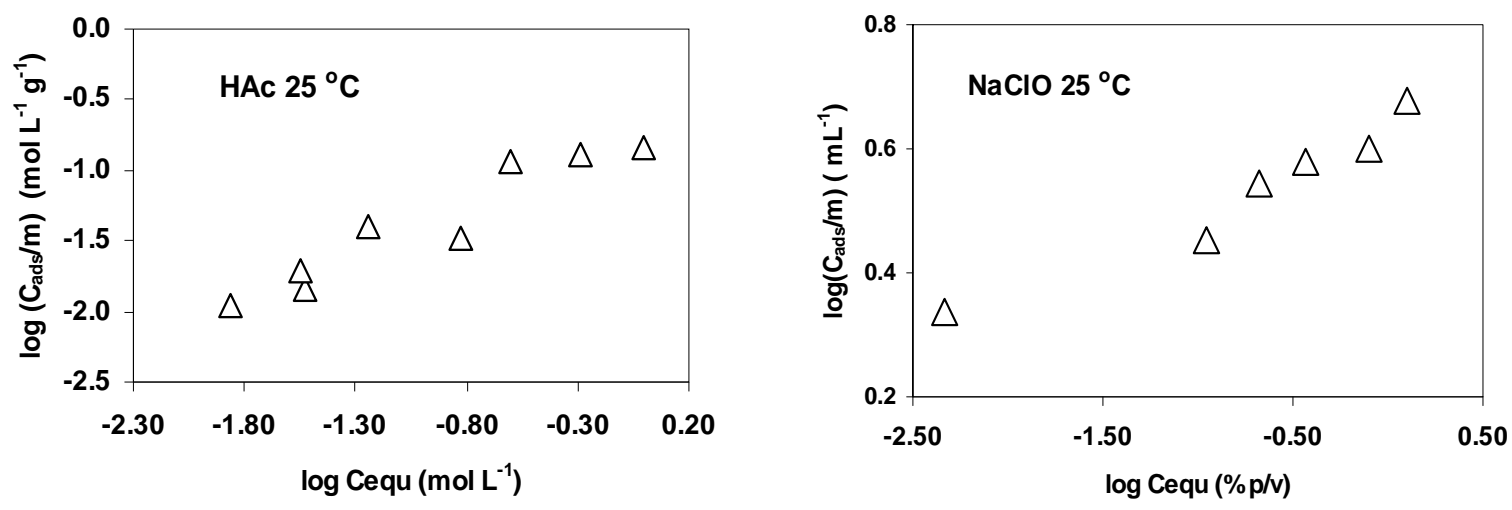

Fig. 2: isotermas de adsorción para HAc y NaClO.

Este modelo propone la existencia de diferentes sitios de adsorción con diferentes energías, lo que indicaría la presencia de cierta heterogeneidad en el carbón activado.

\section{CONCLUSIONES}

El presente trabajo experimental permite que los alumnos adquieran habilidades típicas del laboratorio de química como son la preparación de soluciones, el muestreo y la determinación de la concentración de un soluto por titulación ácido-base y redox. Asimismo, expone al alumno al conocimiento de un programa computacional de cálculo a través del cual, podrá expresar los datos del laboratorio en ecuaciones y realizar gráficos. Los experimentos de cinética y la isoterma de adsorción conducen a la construcción progresiva a través del razonamiento deductivo, de las ideas de equilibrio, tipo de adsorción y de los modelos que describen el fenómeno. Finalmente, el docente puede extrapolar los conocimientos adquiridos a los usos prácticos de la adsorción sobre carbón, como son: la remoción de sustancias colorantes del medio líquido, las máscaras antigás, recuperación y concentración de vitaminas y remoción de otros contaminantes.

\section{REFERENCIAS}

Celsi, S. y Copello, M., Iniciación a la Química Analítica Cuantitativa, $1^{\text {a }}$ edición, El Ateneo, p. 287, Buenos Aires, Argentina (1960).

Cuizano, N., Llanos, B. P., Navarro, A. E, Aplicaciones Ambientales de la Adsorción Mediante Biopolímeros Naturales: Parte 1- Compuestos Fenólicos, Rev. Soc. Quím. Perú 75(4), 495-508 (2009).

D’Alessandro, O., Curia, M. V., Briand, L. E., Cómo Integrar la Ciencia de los Materiales en los Tres Niveles Educativos a través de la Preparación y Observación de Cristales Macroscópicos, Formación Universitaria 2(1), 3-6 (2009).

Dhawale, S. W., Introducing the Treatment of Waste and Wastewater in the General Chemistry Course, Journal of Chemical Education, 70(5), 395-397 (1993).

Jacobsen, E. K., Water Filtration: Small Changes Make a Difference, Journal of Chemical Education, 85(7), 892-893 (2008). 